\title{
Accuracy of Using Mid-Upper Arm Circumference to Detect Wasting Among Children Aged 6-59 Months in Nepal
}

\author{
Krishna Prasad Lamsal, ${ }^{a}$ Kedar Raj Parajuli, ${ }^{b}$ Bhim Kumari Pun, ${ }^{c}$ Ramesh Prasad Adhikari, ${ }^{c}$ Manoj Bashyal, ${ }^{d}$ \\ Baburaja Dangol, ${ }^{\mathrm{e}}$ Kenda Cunningham, ${ }^{\mathrm{c}, \mathrm{f}}$
}

\section{Key Finding}

- Mid-upper arm circumference (MUAC) had a lower sensitivity compared to weight-for-height z-score (WHZ), indicating it can detect only a small proportion of the total number of children aged 6-59 months who are wasted.

\section{Key Implication}

- Using sensitivity and specificity criteria, the poor performance of MUAC compared to WHZ to identify cases of severe and moderate wasting in infants and children aged 6-59 months suggests a need to further refine admission criteria, including the choice of indicators and cutoffs.

\footnotetext{
a Nutritionist, Kathmandu, Nepal.

${ }^{b}$ Nutrition Section, Ministry of Health and Population, Government of Nepal, Kathmandu, Nepal.

${ }^{c}$ Helen Keller International, Kathmandu, Nepal.

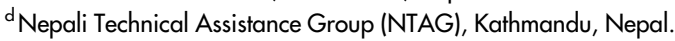

eNewEra, Kathmandu, Nepal.

${ }^{\mathrm{f}}$ London School of Hygiene and Tropical Medicine. London, United Kingdom.

Correspondence to KP Lamsal (lamsal.kp@gmail.com).
}

\section{ABSTRACT}

Background: In countries with a high prevalence of undernutrition, timely, accurate screening at the community level is essential to identify children with wasting. The World Health Organization recommends using either weight-for-height $z$-scores (WHZ) and mid-upper arm circumference (MUAC) or both measures and signs of edema to be used to identify children with severe acute malnutrition for treatment. We compared WHZ and MUAC cutoffs to identify wasting among children aged 6-59 months in Nepal, using $\mathrm{WHZ}$ as the reference standard.

Methods: We used cross-sectional anthropometric data for 3,169 children aged 6-59 months from a 2017 cross-sectional dataset, representative of 42 of Nepal's 77 districts. We used descriptive statistics, receiver operating characteristic (ROC) curves, and kappa statistics to compare the use of MUAC and WHZ to identify wasting. The Youden index was calculated to determine the optimum MUAC cutoffs.

Results: The prevalence of wasting was $3.1 \%$ and $10.5 \%$ using MUAC and $\mathrm{WHZ}$, respectively. We found $13.6 \%$ sensitivity for severe acute malnutrition (SAM) (MUAC $<115 \mathrm{~mm}$ ) and $21.0 \%$ sensitivity for moderate acute malnutrition (MAM) (MUAC $\geq 115$ to $<125 \mathrm{~mm}$ ), with specificity of $99.7 \%$ and $91.2 \%$, respectively. The sensitivity of MUAC for children aged 6-23 months was higher than for children aged 24-59 months. The total area of the ROC curve was 0.53 for the MUAC cutoff for SAM and 0.56 for MAM. The optimum MUAC cutoffs for SAM and MAM were $125 \mathrm{~mm}$ and $132 \mathrm{~mm}$, respectively.

Conclusions: Although MUAC can be used as a rapid screening tool to detect wasting in children aged 6-59 months, using the recommended MUAC cutoffs captures only a small proportion of the total number of wasted children. The poor sensitivity and specificity of MUAC compared to WHZ suggests a need to refine admission and discharge criteria for acute malnutrition management programs to ensure that wasting among infants and children in Nepal is consistently and accurately diagnosed and treated.

\section{INTRODUCTION}

U ndernutrition has devastating individual and public health consequences: it weakens individuals' immune systems, worsens illnesses for individuals, and is linked to poor economic growth and poverty. Furthermore, undernourished children who survive have diminished learning capacity and lower productivity in adulthood. ${ }^{1}$ Acute malnutrition, especially severe acute malnutrition (SAM), is an unstable condition resulting from a relatively short duration of nutritional 


\section{Only using MUAC to screen for malnutrition globally would result in 14.4 of the 16 million children in 2016 with SAM not identified or treated.}

deficit that is often complicated by concurrent infective illness. A severely wasted child has a more than 11 -fold increased risk of death compared to a non-wasted child. ${ }^{2-4}$

In countries with a high prevalence of undernutrition, timely, accurate screening at the community level is essential to identify children with acute malnutrition. More than 500,000 deaths annually could be prevented globally by timely and proper treatment of acute malnutrition. Children aged 6-59 months who have a midupper arm circumference (MUAC) $<115 \mathrm{~mm}$, a weight-for-height z-score (WHZ) $<-3$ standard deviation (SD) (based on the World Health Organization [WHO] Child Growth Standards), and/or nutritional edema are considered to have SAM. Likewise, children aged 6-59 months having WHZ $<-2$ to $\geq-3 \mathrm{SD}$ and/or MUAC $\geq 115 \mathrm{~mm}$ to $<125 \mathrm{~mm}$ are classified as having moderate acute malnutrition (MAM). ${ }^{4}$ MUAC is a widely used tool, especially in resource-limited countries to identify wasting and is closely associated with mortality risk. WHO recommends using either or both WHZ or MUAC and the presence of nutritional edema to assess the prevalence of acute malnutrition and for admission and graduation criteria for treatment programs.

The prevalence of child undernutrition in Nepal is among the highest in the world. Among children aged under 5 years, $10 \%$ are wasted (low weight-for-height) including $2 \%$ severely wasted; this alarmingly high level has persisted for at least 2 decades. ${ }^{6,7}$ To reduce the prevalence of wasting, the Government of Nepal, in collaboration with donors and nongovernmental partners, has been implementing community and health facility-based programs. Since 2009, when the community-based management of acute malnutrition (CMAM) program was piloted, more than 40,000 female community health volunteers have used only MUAC to find wasted children in their communities. In contrast, health facility workers in Nepal use both WHZ and MUAC as well as nutritional edema as criteria to admit children to outpatient therapeutic centers, stabilization centers, and nutrition rehabilitation homes. However, only MUAC is used as the measure for discharge in SAM management programs and as the admission and discharge measure for MAM management programs. ${ }^{7,8}$

Researchers have raised concern about the effectiveness of using MUAC as a wasting screening tool because of its low sensitivity compared to WHZ. $^{9-11}$ As per Fernandez et al. ${ }^{11}$ using MUAC $<115$ mm identified only 10 of 165 children with
WHZ $<-3$. Thus, using MUAC $<115 \mathrm{~mm}$ during community-level screening would leave more than $90 \%$ of children with a WHZ $<-3$ without therapeutic treatment. In other words, only using MUAC to screen for malnutrition globally would result in 14.4 of the 16 million children in 2016 with SAM (defined as WHZ <-3) not identified or treated. Therefore, understanding how MUAC performs, versus WHZ, in the detection of wasting in different populations is important. Prior studies on this have been mostly hospital-based rather than community-based and most were conducted in Africa. To date, no studies have been conducted in Nepal and very few studies done in South Asia to assess the accuracy of MUAC versus WHZ for detection of wasting. Several studies have also raised questions on the appropriateness of using the same cutoff for all children aged 6-59 months, given some findings of age and gender variation. ${ }^{12-15}$ Because WHZ and MUAC are the major anthropometric tools used to identify wasting in children, comparing the accuracy of both tools to identify wasting provides a better sense of the accuracy of measurements. Few studies have been done to compare the diagnostic accuracy of MUAC by assuming WHZ as a gold standard. ${ }^{10-12,15}$ Using WHZ as the reference standard, we aimed to identify the magnitude of discrepancies in the identification of wasted children in Nepal by MUAC versus WHZ, whether these discrepancies vary by child age or gender, and ideal MUAC cutoffs for more thorough detection of children with SAM and MAM.

\section{METHODS}

This study uses a cross-sectional dataset collected by a local survey firm in July-September 2017 across 16 of Nepal's 77 districts, including a total sample of 3,169 children aged 6-59 months. Structured questionnaires were used to interview the young child's mother and a household head (with preference given to a male decision maker, when available) to obtain socioeconomic and demographic information, as well as to measure knowledge and practices related to nutrition, reproductive health and family planning, agriculture and food security, empowerment, and exposure to health and nutrition-related interventions.

Anthropometry, including length/height, weight, and MUAC of each child, was measured by a team of 20 trained and standardized data collectors. The length of children aged 6-23 months and height of children aged 24-59 months were measured using the vertical stadiometer, following WHO guidelines. ${ }^{16}$ Weight was measured with an electronic digital 
weighing scale (Seca scale) and read to the nearest $0.1 \mathrm{~kg}$ with minimum light clothing. Calibration of the Seca scales was done before weighing each child. For MUAC measurement, we used nonstretchable and flexible MUAC tapes procured from United Nations Children's Fund. The measurement was taken at the midpoint of the acromion of the scapula and the olecranon of the ulna of a child. The height/length and MUAC of each child were measured twice and the measurements averaged to get the final raw length/height, weight, and MUAC measurements.

These anthropometric data were then transformed into WHZ z-scores. The prevalence of wasting was calculated by using both WHZ and MUAC. To classify children as SAM and MAM using WHZ, we used cutoffs of $<-3$ SD WHZ and $\geq-3$ to $<-2$ SD WHZ, respectively. To classify children as SAM and MAM using MUAC, we used cutoffs of $<115 \mathrm{~mm}$ and $\geq 115 \mathrm{~mm}$ to $<125 \mathrm{~mm}$, respectively. ${ }^{4,7}$ We assumed WHZ as the reference measure and tested the sensitivity of MUAC cutoffs versus WHZ. The sensitivity and false-positive rates (1-specificity) of MUAC were determined using WHO classification for SAM and MAM. To assess the performance of MUAC cutoffs compared to the standard recommended by WHO to define SAM and MAM, receiver operating characteristic (ROC) curves were constructed and categorized as: $50 \%-60 \%$ very poor, $61 \%-70 \%$ poor, $71 \%-80 \%$ fair, $81 \%-$ $90 \%$ good, and $91 \%-100 \%$ with excellent sensitivity. The ROC curve is the plot of sensitivity versus false-positive rate of MUAC cutoffs. The area under the curve (AUC) is the area between the curve and the segment $(0,0)$ and $(1,1)$, which corresponds to a random classifier. A larger AUC indicates a more accurate diagnosis of acute malnutrition, defined by WHZ cutoffs. ${ }^{5,10,17,18}$ Kappa statistic was used to analyze inter-rater agreement between MUAC and WHZ by assuming: $<0 \%$ as none, $0 \%-20 \%$ as poor, $21 \%-40 \%$ as slight, $41 \%-60 \%$ as fair, $61 \%-80 \%$ as good, $81 \%-92 \%$ as very good, and $93 \%$ or more as excellent agreement. ${ }^{19}$ To identify the MUAC cutoff with the highest sensitivity and specificity, the Youden index, which is the difference between the true-positive rate (sensitivity) and the false-positive rate, was calculated. ${ }^{20}$ All analyses were done using STATA (Version 15).

\section{Ethical Approval}

We obtained ethical clearance from the Ministry of Health and Population, Nepal Health Research Council, Kathmandu, Nepal (NHRC \#1620/2017). All respondents gave written informed consent before the interview and collection of anthropometric data. No treatment or incentive was given to the study participants.

\section{RESULTS}

Selected background characteristics of the surveyed households and children aged 6-59 months are presented in Table 1. Children were on average aged 28 months; slightly less than half $(43.7 \%)$ were aged 6-23 months, and the rest (56.2\%) were aged 24-59 months. More than half $(55.8 \%)$ of the children were male, and more than half $(56.5 \%)$ resided in the hilly region of the country, versus the lowland plains and mountains of Nepal. Almost half $(49.6 \%)$ of the surveyed children were from a socially excluded ethnic group. Among the children, $28.0 \%$ were stunted (low height-for-age). The overall prevalence of wasting based on WHZ was $10.5 \%$, comprised of $2.1 \%$ SAM and $8.5 \%$ MAM, whereas only $0.4 \%$ SAM and $2.7 \%$ MAM were found based on MUAC.

We found $13.6 \%$ sensitivity for SAM (MUAC $<115 \mathrm{~mm})$ and $21.0 \%$ sensitivity for MAM (MUAC $\geq 115 \mathrm{~mm}$ to $<125 \mathrm{~mm}$ ) with specificity of $99.7 \%$ and $91.2 \%$, respectively. The total area of the ROC curve was 0.53 for the MUAC cutoff for SAM $(<115 \mathrm{~mm})$. The kappa value for the SAM cutoff was $9.0 \%$. The total area under ROC curve was 0.56 for the MAM cutoff $\geq 115 \mathrm{~mm}$ to $<125 \mathrm{~mm}$. The kappa value for the MAM cutoff was $17.0 \%$.

Comparison of ROC and kappa by child gender and age are shown in Table 2. The total AUC of the SAM cutoff for male and female children were 0.51 and 0.56 , respectively. The kappa value for the SAM cutoff among boys was $4.0 \%$ and $16.0 \%$ for girls. The total AUC of the MAM cutoff for boys and girls was 0.52 and 0.62 with kappa values $11.0 \%$ and $24.0 \%$, respectively.

Among the children aged 6-23 months, 11.5\% were found wasted including $8.9 \%$ with MAM and $2.6 \%$ with SAM using WHZ, but only 5.0\% MAM and $0.7 \%$ SAM were identified as wasted using MUAC. Among children aged 24-59 months 9.8\% were identified as wasted using WHZ including $8.2 \%$ with MAM and $1.6 \%$ with SAM, whereas using MUAC, only $0.7 \%$ and $0.2 \%$ of these children were diagnosed with MAM and SAM, respectively. The total AUC of the SAM cutoff was 0.61 and 0.51 for children aged 6-23 months and 2459 months, respectively. The kappa value of the SAM cutoff for children aged 6-23 months was $12.0 \%$ and $6.0 \%$ for children aged 24-59 months. Likewise, the total AUC of the MAM cutoff for children aged 6-23 months and 24-59 months were

\section{Among the children aged 6- 23 months, 8.9\% with MAM and $2.6 \%$ with SAM were identified as wasted using WHZ, but only $5.0 \%$ with MAM and $0.7 \%$ with SAM were identified as wasted using MUAC.}


TABLE 1. Background Characteristics of Children Aged 6-59 Months Surveyed on Undernutrition, Nepal

\begin{tabular}{|c|c|}
\hline & No. $(\%), N=3,169$ \\
\hline \multicolumn{2}{|l|}{ Sex } \\
\hline Male & $1,768(55.8)$ \\
\hline Female & $1,401(44.2)$ \\
\hline \multicolumn{2}{|l|}{ Child age, months } \\
\hline 6-23 & $1,387(43.7)$ \\
\hline $24-59$ & $1,782(56.2)$ \\
\hline \multicolumn{2}{|l|}{ Ecological zone } \\
\hline Mountain & $389(12.3)$ \\
\hline Hill & $1,790(56.5)$ \\
\hline Terai & $990(31.2)$ \\
\hline \multicolumn{2}{|l|}{ Type of residence } \\
\hline Urban & $1,588(50.1)$ \\
\hline Rural & $1,581(49.9)$ \\
\hline \multicolumn{2}{|l|}{ Caste/ethnicity } \\
\hline Socially excluded & $1,571(49.6)$ \\
\hline Brahmin/Chhetri & $1,247(39.3)$ \\
\hline Others & $351(11.1)$ \\
\hline \multicolumn{2}{|l|}{ Equity quintile } \\
\hline Lowest & $681(21.5)$ \\
\hline 2nd lowest & $906(28.6)$ \\
\hline Middle & $745(23.5)$ \\
\hline 2nd highest & $638(20.1)$ \\
\hline Highest & $199(6.3)$ \\
\hline \multicolumn{2}{|l|}{ Mother's education level } \\
\hline Never attended school & $693(21.8)$ \\
\hline Some primary school & $439(13.8)$ \\
\hline Completed grade 5 & $243(7.7)$ \\
\hline Some secondary education & $1,051(33.2)$ \\
\hline Completed secondary education & $392(12.4)$ \\
\hline Completed class 12 & $289(9.1)$ \\
\hline Higher education & $62(1.9)$ \\
\hline \multicolumn{2}{|l|}{ Prevalence of food insecurity } \\
\hline Food secure & $2,135(67.4)$ \\
\hline Mildly food insecure & $497(15.7)$ \\
\hline Moderately food insecure & $465(14.7)$ \\
\hline Severely food insecure & $71(2.2)$ \\
\hline WHZ & \\
\hline
\end{tabular}

Continued
TABLE 1. Continued

No. $(\%), N=3,169$

\begin{tabular}{ll}
\hline SAM $(<-3$ SD) & $65(2.1)$ \\
\hline MAM $<-2$ SD to $\geq-3$ SD) & $269(8.5)$ \\
\hline Normal $(>-2$ SD) & $2,835(89.4)$ \\
\hline MUAC & $12(0.4)$ \\
\hline SAM $(<115 \mathrm{~mm})$ & $87(2.7)$ \\
\hline MAM $(\geq 115 \mathrm{~mm}$ to $<125 \mathrm{~mm})$ & $3070(96.9)$ \\
\hline Normal $(>125 \mathrm{~mm})$ & \\
\hline Stunting & $888(28)$ \\
\hline Stunted & $2,279(72)$ \\
\hline Not stunted (Normal) & \\
\hline
\end{tabular}

Abbreviations: MAM, moderate acute malnutrition; MUAC, midupper arm circumference; SAM, severe acute malnutrition; SD, standard deviation; WHZ, weight-for-height/length z-score.

0.61 and 0.53 , respectively. The kappa value of the MAM cutoff for children aged 6-23 months was $25.1 \%$ and $7.0 \%$ for children aged $24-59$ months.

Table 3 shows the sensitivity, specificity, and Youden index at various MUAC cutoff values for diagnosing SAM. The optimum cutoff of MUAC for SAM was found to be $125 \mathrm{~mm}$ with a maximum Youden index of $49.9 \%$. Similarly, the best cutoff point of MUAC for optimum diagnosis of MAM was found to be $132 \mathrm{~mm}$ with a Youden index $30.5 \%$ (Table 4 ).

\section{DISCUSSION}

This study compares the sensitivity and specificity of MUAC versus WHZ to identify wasting in children aged 6-59 months in Nepal, to contribute to local and global debates on how to ensure the timely and accurate diagnosis of wasting, a prerequisite to identifying and treating millions of children globally suffering from undernutrition. This is the first-ever study comparing the performance of MUAC and WHZ in Nepal to identify wasted children and among the few studies globally to use population-based, rather than hospital/treatment center-based, data to explore variations in the MUAC and WHZ indicators. This study looks at discrepancies in classification overall and then separately by child age and child gender. The percentage of wasting, as measured by WHZ, was found to be almost the same as the national prevalence found in the recent demographic and 
TABLE 2. Area Under ROC Curve and Kappa Value to Compare MUAC and WHZ Cutoffs for Children Aged 6-59 Months, Nepal

\begin{tabular}{|c|c|c|c|c|}
\hline & \multicolumn{2}{|c|}{$\operatorname{SAM}(<115 \mathrm{~mm})$} & \multicolumn{2}{|c|}{$\operatorname{MAM}(\geq 115$ to $<125 \mathrm{~mm})$} \\
\hline & Area Under ROC & Kappa, \% & Area Under ROC & Kappa, \% \\
\hline Overall & 0.53 & 9 & 0.56 & 17 \\
\hline \multicolumn{5}{|l|}{ Sex } \\
\hline Male & 0.51 & 4 & 0.52 & 11 \\
\hline Female & 0.56 & 16 & 0.62 & 24 \\
\hline \multicolumn{5}{|c|}{ Child age, months } \\
\hline $6-23$ & 0.61 & 12 & 0.61 & 25 \\
\hline $24-59$ & 0.51 & 6 & 0.53 & 7 \\
\hline \multicolumn{5}{|l|}{ Stunting } \\
\hline Stunted & 0.51 & 10 & 0.56 & 16 \\
\hline Not stunted & 0.54 & 10 & 0.56 & 16 \\
\hline
\end{tabular}

Abbreviations: MAM, moderate acute malnutrition; MUAC, mid-upper arm circumference; ROC, receiver operating characteristic curve; SAM, severe acute malnutrition; WHZ, weight-for-height/length z-score.

health survey done in 2016 (10.5\% vs. 9.8\%). ${ }^{6}$ Among these wasted children, we found that $2.1 \%$ were SAM and $8.5 \%$ were MAM, but when using MUAC we only found $0.4 \%$ SAM and $2.7 \%$ MAM. When comparing the 2 methods for identification, and using WHZ as the reference standard, the total AUC of MUAC for both SAM and MAM, showed that in $53 \%$ and $56 \%$, respectively. These are close to an AUC of 0.5, which is the same as complete randomness, and suggests that the current MUAC cutoffs are poor tools for detecting wasting.

With WHZ as the reference standard, we found MUAC only had $13.6 \%$ sensitivity for SAM (MUAC $<115 \mathrm{~mm}$ ) and $21 \%$ sensitivity for MAM (MUAC $\geq 115 \mathrm{~mm}$ to $<125 \mathrm{~mm}$ ), with a specificity of $99.7 \%$ and $91.2 \%$, respectively. This is consistent with various studies that have reported a very wide range of sensitivity of MUAC, ranging from $17.5 \%$ to $43.5 \%$ and consistently higher specificity. ${ }^{6,9-11,21}$ A study by Grellety et al. ${ }^{21}$ in a therapeutic feeding center that included 2,205 South Sudanese children concluded that MUAC $<115 \mathrm{~mm}$ would have failed to identify $33.0 \%$ of the children with SAM, while $98.0 \%$ were identified by WHZ $<-3$ SD alone and $100 \%$ by MUAC $<130 \mathrm{~mm}$. A systematic review and meta-analysis by Grellety and Golden $^{22}$ concluded that the use of MUAC alone to identify SAM children, versus using both WHZ and MUAC, would exclude many children from treatment and thus, result in an additional 300,000 SAM related deaths annually. In Asia, the evidence around these challenges with using MUAC to identify and treat malnourished children is building. Fiorentino et al. ${ }^{23}$ also found in Cambodia that the sensitivity of MUAC ranged from $6.5 \%$ to $32.9 \%$ in children with acute malnutrition and from $0.0 \%$ to $18.2 \%$ in children with severe acute malnutrition. According to Talapalliwar and Garg $^{24}$ the sensitivity and specificity of MUAC $<115 \mathrm{~mm}$ was $13.6 \%$ and $99.3 \%$, respectively and the current cutoff can only capture a small proportion of all children with SAM, in the context of tribal populations in India. Our findings also show that many Nepalese children could be excluded by using only MUAC to identify wasting.

Similarly, since MUAC $>115 \mathrm{~mm}$ is being used in Nepal as a single criterion to discharge children with SAM from outpatient therapeutic centers, proper nutritional rehabilitation will be missed, and thousands of Nepalese children will be discharged as cured who could still be suffering from undernutrition or at increased risk of relapse. Finally, many studies to date focus on SAM, but MAM management programs, especially blanket and targeted supplementary feeding programs in Nepal, use MUAC only. These findings show that a large number of children with MAM are being missed and not provided with nutrition rehabilitation, counseling, and support because MUAC was the only anthropometric measurement used. Existing literature suggests that MUAC-only based programs tend to identify significantly more girls and younger children than those identified by

\section{These findings show that a large number of children with MAM are being missed and not provided with nutrition rehabilitation, counseling, and support because MUAC was the only anthropometric measurement used.}


TABLE 3. Sensitivity, Specificity, and Youden Index at Various Cutoffs of MUAC for SAM in Children Aged 659 Months, Nepal

\begin{tabular}{|c|c|c|c|}
\hline MUAC, mm & Sensitivity, \% & Specificity, \% & Youden Index, \% \\
\hline 105 & 4.0 & 100 & 4.0 \\
\hline 106 & 4.1 & 100 & 4.1 \\
\hline 107 & 4.8 & 100 & 4.8 \\
\hline 108 & 5.1 & 100 & 5.1 \\
\hline 109 & 5.4 & 100 & 5.4 \\
\hline 110 & 6.9 & 100 & 6.9 \\
\hline 111 & 7.6 & 100 & 7.6 \\
\hline 112 & 9.1 & 100 & 9.1 \\
\hline 113 & 11.2 & 100 & 11.2 \\
\hline 114 & 12.8 & 99.8 & 12.6 \\
\hline 115 & 13.6 & 99.7 & 13.3 \\
\hline 116 & 14.3 & 99.0 & 13.3 \\
\hline 117 & 18.9 & 99.1 & 18.0 \\
\hline 118 & 21.6 & 98.7 & 20.3 \\
\hline 119 & 24.3 & 98.5 & 22.8 \\
\hline 120 & 28.9 & 98.3 & 27.2 \\
\hline 121 & 34.5 & 98.1 & 32.6 \\
\hline 122 & 35.9 & 98.0 & 33.9 \\
\hline 123 & 43.6 & 96.6 & 40.2 \\
\hline 124 & 46.8 & 96.4 & 43.2 \\
\hline 125 & 53.7 & 96.2 & 49.9 \\
\hline 126 & 59.0 & 87.2 & 46.2 \\
\hline 127 & 66.0 & 73.0 & 39.0 \\
\hline 128 & 69.0 & 56.3 & 35.3 \\
\hline 129 & 72.0 & 46.1 & 31.8 \\
\hline 130 & 90.1 & 41.0 & 31.1 \\
\hline 131 & 93.0 & 37.5 & 30.5 \\
\hline 132 & 93.5 & 33.3 & 26.8 \\
\hline 133 & 93.1 & 31.5 & 24.6 \\
\hline 134 & 93.2 & 28.5 & 21.7 \\
\hline 135 & 97.6 & 26.2 & 23.8 \\
\hline
\end{tabular}

Abbreviations: MUAC, mid-upper arm circumference; SAM, severe acute malnutrition.

WHZ. ${ }^{23}$ In this study, the total AUC for male children was slightly lower than for female children (0.51 vs. 0.56), with kappa $4.0 \%$ and $16.0 \%$, respectively, which indicates that MUAC captured faintly more wasted female children than male children. The ROC and kappa results in this study also suggest that MUAC may be a more useful approach for the diagnosis of both SAM and MAM in children aged less than 2 years than for older children. Prior studies also provide similar evidence of age variation in the ability of MUAC to detect both SAM and MAM. ${ }^{23,25-27}$ The age dependency may be due to variation of muscle mass with increasing age of children. 
TABLE 4. Sensitivity, Specificity, and Youden Index at Various Cutoffs of MUAC for MAM in Children Aged 659 Months, Nepal

\begin{tabular}{|c|c|c|c|}
\hline MUAC, mm & Sensitivity, \% & Specificity, \% & Youden Index, \% \\
\hline 115 & 0.2 & 100 & 0.2 \\
\hline 116 & 0.6 & 100 & 0.6 \\
\hline 117 & 1.2 & 100 & 1.2 \\
\hline 118 & 3.4 & 100 & 3.4 \\
\hline 119 & 3.9 & 99.5 & 3.4 \\
\hline 120 & 5.8 & 98.0 & 3.8 \\
\hline 121 & 9.4 & 97.1 & 6.5 \\
\hline 122 & 12.0 & 96.4 & 8.4 \\
\hline 123 & 14.3 & 95.2 & 9.5 \\
\hline 124 & 17.0 & 94.1 & 11.1 \\
\hline 125 & 21.0 & 91.2 & 12.2 \\
\hline 126 & 33.2 & 87.3 & 20.5 \\
\hline 127 & 39.2 & 84.3 & 23.5 \\
\hline 128 & 45.2 & 80.4 & 25.6 \\
\hline 129 & 51.2 & 76.8 & 28.0 \\
\hline 130 & 61.2 & 69.3 & 29.5 \\
\hline 131 & 64.3 & 65.4 & 29.7 \\
\hline 132 & 66.6 & 63.9 & 30.5 \\
\hline 133 & 68.9 & 57.0 & 25.9 \\
\hline 134 & 71.2 & 51.0 & 22.2 \\
\hline 135 & 71.6 & 49.2 & 20.8 \\
\hline 136 & 74.3 & 46.3 & 20.6 \\
\hline 137 & 75.8 & 44.2 & 20.0 \\
\hline 138 & 77.6 & 40.1 & 17.7 \\
\hline 139 & 79.6 & 37.5 & 17.1 \\
\hline 140 & 81.6 & 34.5 & 16.1 \\
\hline
\end{tabular}

Abbreviations: MAM, moderate acute malnutrition; MUAC, mid-upper arm circumference.

In line with previous studies, ${ }^{25,26,28}$ the results of our Youden index analyses, which identify an ideal performance point by calculating the optimum level of sensitivity and specificity, suggest that current MUAC cutoffs must be increased to capture more cases of wasting. The optimum MUAC cutoff point to identify children with SAM was found to be $125 \mathrm{~mm}$. Likewise, the best cutoff for MUAC to optimally identify children with MAM was found to be $132 \mathrm{~mm}$. Talapalliwar and Garg $^{24}$ also found the optimal cutoff of MUAC to be $125 \mathrm{~mm}$ for proper detection of SAM and $136 \mathrm{~mm}$ for diagnosing MAM. Laillou et al. ${ }^{5}$ suggested that a MUAC cutoff of $133 \mathrm{~mm}$ would allow the inclusion of more than $65 \%$ of children with a WHZ $<-3$ to be considered wasted. Similarly, a recent study by Tessema et al. ${ }^{29}$ in Ethiopia concluded that implementation of a MUAC-only screening program, using a cutoff of $<115 \mathrm{~mm}$ for the identification of SAM, is unethical as it may lead to many children remaining undiagnosed and untreated. They suggested a MUAC cutoff $<133 \mathrm{~mm}$ for optimum identification of children with SAM. Furthermore, consistent with our findings, Fiorentino et al. ${ }^{23}$ concluded that MUAC cutoffs by age group and 
gender should be revised for community-level screening and treatment of wasting.

\section{Limitations}

A limitation of this study is that we could not analyze relationships between the screening tools and mortality, screening tools and response to treatment such as weight gain, or time for nutrition rehabilitation/graduation from treatment. We have used a cross-sectional dataset, which could not capture information on response to treatment or recovery/survival. Furthermore, screening and exclusion of children with edema were not done in these analyses, which could offer yet another interesting perspective on the prevalence of acute malnutrition. We used WHZ as a reference standard, but WHZ itself is not without its challenges for diagnosis and treatment of wasting, including the need for all health workers to be trained and standardized to ensure accuracy of measurement and for anthropometric equipment to be maintained in good condition.

\section{CONCLUSION}

This study confirms that MUAC, using WHZ as the reference standard, can detect wasting but in only a small fraction of all wasted children, leaving the majority of wasting in Nepal undetected and untreated. The poor performance in terms of sensitivity and specificity confirms the need to either increase the MUAC cutoff values or adopt both MUAC and WHZ at every health facility and in acute malnutrition management programs for early, rapid, and accurate diagnosis and treatment of wasting in Nepal. Therefore, WHZ should also be used as the admission and discharge criteria, rather than MUAC alone, as a standalone anthropometric criterion at therapeutic centers. Additionally, the Government of Nepal and development partners will need to continue to work with communities to ensure that more children are brought to the health facilities where these criteria would be used. Furthermore, while evidence-based updating is done to policies and programs for the treatment of undernutrition, governments and development partners should continue to invest in prevention efforts. Multisectoral policies and programs focused on the prevention of all forms of child malnutrition should continue so that the prevalence of wasting does not remain above $10 \%$ for another 20 years. The findings of this study can help the Government of Nepal and development partners to update screening and management approaches for acute malnutrition to enable timely and proper nutrition rehabilitation of children aged under 5 years.

Acknowledgments: The authors also acknowledge Dr. Bernadette Cichon at No Wasted Lives/Action Against Hunger, for her feedback on an early draft of the manuscript.

Funding: This publication was prepared using data from Suaahara II, funded by the United States Agency for International Development (USAID) under a Cooperative Agreement (No. AID-367-A-16-00006) between USAID and Helen Keller International.

Disclaimer: The contents of this publication are the sole responsibility of the authors and do not necessarily reflect the views of USAID or the United States Government.

Author contributions: KPL and KJC jointly conceptualized the study. KPL and RA did the data analysis. KPL wrote the first draft of the manuscript. KRP reviewed the manuscript as a focal person of the Government of Nepal. BD, BP, and MB reviewed the anthropometric data. KJC did the final review of the manuscript. All authors participated in the interpretation of the data and critical revisions of the manuscript. All authors read and approved the final manuscript.

Competing interests: None declared.

\section{REFERENCES}

1. Black RE, Allen LH, Bhutta ZA, et al. Maternal and child undernutrition: global and regional exposures and health consequences. Lancet. 2008;371(9608):243-260. CrossRef. Medline

2. Olofin I, McDonald CM, Ezzati M, et al. Associations of suboptimal growth with all-cause and cause-specific mortality in children under five years: a pooled analysis of ten prospective studies. PLoS One. 2013;8(5):e64636. CrossRef. Medline

3. Collins S. Treating severe acute malnutrition seriously. Arch Dis Child. 2007;92(5):453-461. CrossRef. Medline

4. World Health Organization (WHO). Guideline: Updates on the Management of Severe Acute Malnutrition in Infants and Children. WHO; 2013. Accessed November 5, 2021. https://www.who.int/ publications/i/item/9789241506328

5. Laillou A, Prak S, de Groot R, et al. Optimal screening of children with acute malnutrition requires a change in current $\mathrm{WHO}$ guidelines as MUAC and WHZ identify different patient groups. PLoS One. 2014;9(7):e101159. CrossRef. Medline

6. Government of Nepal. Ministry of Health $(\mathrm{MOH})$; New ERA; ICF. Nepal Demographic and Health Survey 2016. MOH; 2017. Accessed November 5, 2021. http://dhsprogram.com/pubs/pdf/ FR336/FR336.pdf

7. Subedi G, Sharma A, Lamsal KP, Sharma B, Thapa D. IMAM Training Manual. Ministry of Health, Nepal; 2016.

8. United Nations Children's Fund (UNICEF). Evaluation of Community Management of Acute Malnutrition (CMAM): Nepal Country Case Study. UNICEF; 2012. Accessed November 5, 2021. https://assets. publishing.service.gov.uk/media/57a08a6140f0b6497400 0584/60997-Nepal-CMAM-evaluation-UNICEF-1 loct2012. pdf

9. Berkley J, Mwangi I, Griffiths K, et al. Assessment of severe malnutrition among hospitalized children in rural Kenya: comparison of weight for height and mid upper arm circumference. JAMA. 2005;294(5):591-597. CrossRef. Medline

10. Dairo MD, Fatokun ME, Kuti M. Reliability of the mid upper arm circumference for the assessment of wasting among children aged 1259 Months in Urban Ibadan, Nigeria. Int J Biomed Sci. 2012; 8(2): 140-143. Medline 
11. Fernández MAL, Delchevalerie $P$, van Herp M. Accuracy of MUAC in the detection of severe wasting with the new WHO growth standards. Pediatrics. 2010;126(1):e195-e201. CrossRef. Medline

12. Briend A, Alvarez J-L, Avril N, et al. Low mid-upper arm circumference identifies children with a high risk of death who should be the priority target for treatment. BMC Nutrition. 2016/10/21 2016;2 (1):63. CrossRef

13. Hall G, Chowdhury S, Bloem M. Use of mid-upper-arm circumference $Z$ scores in nutritional assessment. Lancet. 1993;341 (8858): 1481. CrossRef. Medline

14. Ulijaszek SJ, Kerr DA. Anthropometric measurement error and the assessment of nutritional status. Br J Nutr. 1999;82(3):165-177. CrossRef. Medline

15. Hop T, Gross R, Sastroamidjojo S, Giay T, Schultink W. Mid-upperarm circumference development and its validity in assessment of undernutrition. Asia Pac J Clin Nutr. 1998;7(1):65-69. Medline

16. World Health Organization (WHO). Department of Nutrition for Health and Development. Training Course on Child Growth Assessment: WHO Child Growth Standards. Course Director's Guide. WHO; 2008. https://www.who.int/childgrowth/training/ module_h_directors_guide.pdf

17. Hajian-Tilaki K. Receiver operating characteristic (ROC) curve analysis for medical diagnostic test evaluation. Caspian J Intern Med. 2013;4(2):627-635. Medline

18. Park SH, Goo JM, Jo CH. Receiver operating characteristic (ROC) curve: practical review for radiologists. Korean J Radiol. 2004;5 (1):11-18. CrossRef. Medline

19. Carpenter CR. Kappa statistic. CMAJ. 2005;173(1):15-26; author reply 17. CrossRef. Medline

20. Youden WJ. Index for rating diagnostic tests. Cancer. 1950;3(1):3235. CrossRef. Medline

21. Grellety E, Krause LK, Shams Eldin M, Porten K, Isanaka S. Comparison of weight-for-height and mid-upper arm circumference (MUAC) in a therapeutic feeding programme in South Sudan: is MUAC alone a sufficient criterion for admission of children at high risk of mortality? Public Health Nutr. 2015;18(14):2575-2581. CrossRef. Medline

22. Grellety E, Golden MH. Severely malnourished children with a low weight-for-height have a higher mortality than those with a low midupper-arm-circumference: I. Empirical data demonstrates Simpson's paradox. Nutr J. 2018;17(1):79. CrossRef. Medline

23. Fiorentino M, Sophonneary P, Laillou A, et al. Current MUAC cut-offs to screen for acute malnutrition need to be adapted to gender and age: the example of Cambodia. PLoS One. 2016;1 1(2):e0146442. CrossRef. Medline

24. Talapalliwar M, Garg B. Diagnostic accuracy of mid-upper arm circumference (MUAC) for detection of severe and moderate acute malnutrition among tribal children in central India. Int J Med Sci Public Health. 2016;5(7):1317. CrossRef

25. Custodio E, Martin-Cañavate R, Di Marcantonio F, Molla D, Abukar $Y$, Kayitakire F. MUAC-for-age more useful than absolute MUAC for nutritional surveillance in Somalia: results from nineteen cross sectional surveys (2007-2016). BMC Nutrition. 2018;4(1):8. CrossRef

26. Habibzadeh F, Habibzadeh P, Yadollahie M. On determining the most appropriate test cut-off value: the case of tests with continuous results. Biochem Med (Zagreb). 2016;26(3):297-307. CrossRef. Medline

27. Sougaijam R, Gupta SS, Raut AV, Bharambe MS, Garg BS. Validating the MUAC (mid-upper arm circumference) cut-off for detection of severe acute malnutrition in children aged 6-59 months in rural Maharashtra. Indian Pediatr. 2019;56(3):209-212. Medline

28. Marshall SK, Monárrez-Espino J, Eriksson A. Performance of midupper arm circumference to diagnose acute malnutrition in a crosssectional community-based sample of children aged 6-24 months in Niger. Nutr Res Pract. 2019;13(3):247-255. CrossRef. Medline

29. Tessema M, Laillou A, Tefera A, Teklu Y, Berger J, Wieringa FT. Routinely MUAC screening for severe acute malnutrition should consider the gender and age group bias in the Ethiopian non-emergency context. PLoS One. 2020;15(4):e0230502. CrossRef. Medline

\section{Peer Reviewed}

Received: August 6, 2021; Accepted: October 5, 2021; First published online: December 9, 2021.

Cite this article as: Lamsal KP, Parajuli KR, Pun BK, et al. Accuracy of using mid-upper arm circumference to detect wasting among children aged 6-59 months in Nepal. Glob Health Sci Pract. 2021;9(4):881-889. https://doi.org/10.9745/GHSP-D-20-00450

(C) Lamsal et al. This is an open-access article distributed under the terms of the Creative Commons Attribution 4.0 International License (CC BY 4.0), which permits unrestricted use, distribution, and reproduction in any medium, provided the original author and source are properly cited. To view a copy of the license, visit https://creativecommons.org/licenses/by/4.0/. When linking to this article, please use the following permanent link: https:// doi.org/10.9745/GHSP-D-20-00450 